\title{
Perspectives of Syrian Refugees on Antibiotic Use and Prescribing in Dutch Primary Care: A Qualitative Study
}

Rima Alkirawan ( $\nabla$ rimakirawan@hotmail.com )

VUMC https://orcid.org/0000-0002-2666-3190

Ramin Kawous

Pharos Centre of Expertise on Health Disparities

Evert Bloemen

Pharos Centre of Expertise on Health Disparities

Maria E.T.C. van den Muijsenbergh

Radboud Universiteit

Simone Goosen

universitiet van Amsterdam

Fijgje de Boer

VU medisch centrum

Research article

Keywords: antibiotic use, primary care, migration, the Netherlands

Posted Date: August 25th, 2020

DOI: https://doi.org/10.21203/rs.3.rs-56378/v1

License: (9) This work is licensed under a Creative Commons Attribution 4.0 International License.

Read Full License

Version of Record: A version of this preprint was published at International Journal of Migration, Health and Social Care on April 12th, 2022. See the published version at https://doi.org/10.1108/IJMHSC-122021-0112. 


\section{Abstract}

Background: Antibiotic resistance is an international public health concern. Medical culture influences antibiotic use. Migrants, like Syrian refugees, are confronted with a different health care system in their new country and also with different culture regarding antibiotic prescription. The aim of this study is to get insight into the perspectives, knowledge and practices among Syrian refugees regarding antibiotic use and prescribing in Dutch primary care.

Methods: A qualitative study using semi-structured interviews with 12 Syrian refugees living in the Netherlands was conducted.

Results: participants stressed the easy access to antibiotics in Syria and reported storing antibiotics at home for emergencies. In the Netherlands, some of them still self-prescribed antibiotics while others adhered to the Dutch GP's advice. Syrian refugees believed in having a weaker immunity compared to the Dutch inhabitants. For their young children, they adhered to the new health care system which resulted in not giving the children antibiotics without a prescription. Several complaints about the Dutch health care system were identified such as difficult access to the desired medication and lack of medical care for their complaints. Most respondents preferred the Syrian health care system.

Conclusion: Syrian refugees experienced restricted access to antibiotics in Dutch primary care which was contrary to their experiences in Syria. As a reaction, they continued self-medicating with antibiotics. However, some of them adapted to the Dutch health care culture and accepted the alternative treatment proposal. For their children most participants adhered to the prescription policy of antibiotics in the Netherlands.

\section{Background}

The increase of antibiotic resistance is a worldwide public health threat [1] which according to The World Health Organization (WHO) affects all populations in the world and is difficult to constrain within individual countries $[2,3]$. There is a worldwide consensus on the need for rationalizing antibiotic consumption in order to combat antibiotic resistance [2]. In order to reduce the spread of this universal phenomenon, WHO launched a global campaign to facilitate the optimal use of antibiotics [2]. Countries around the world have different medical cultures and taking medication is always influenced by the medical culture [4]. Cultural health beliefs could be a factor that explains the considerable variation in antibiotic use among countries [5]. Differences in cultural health beliefs become visible when a person is confronted with a health care system that differs from the one with which a person is familiar. For instance, migrants from Middle Eastern countries living in Scandinavian countries criticized the treatment strategies of illnesses and the role of the general practitioner who in their perception behaved as an advisor and not as a real doctor [6]. Discontent was also shown with the long process before obtaining access to a specialist, which is completely different from what they are used to in their homelands, where private clinics are available around the clock [6]. 
In recent years, the Syrians have become one of the main migrant populations with a non-western background in the Netherlands within a short period of time [7]. Consequently, Syrian refugees were confronted with a health care system that differs on several aspects to the one they are used to in their home country. Next to being confronted with primary care services for the first time in their lives [8, 9], less antibiotics are prescribed and less over the counter medication can been obtained in the Netherlands compared to Syria. In line with Dutch guidelines, general practitioners apply restricted indications for the prescription of antibiotics [10]. In the Netherlands, the least amount of antibiotics is prescribed compared to other European countries [11]. The contrast with Syria is huge where the absence of general practitioners, unnecessary prescribing by physicians, lack of treatment guidelines and over the counter sales of antibiotics by pharmacists contribute to high antibiotic use and abuse in Syria [12]. In order to rationalize antibiotic prescribing, it is important for Dutch GPs to have knowledge about patient's expectations in terms of antibiotic treatment [13], since non-prescription would feed distrust in the health provider [14].

This study aimed to explore the perspectives and expectations of Syrian refugees in the Netherlands about antibiotic use and prescribing in Dutch primary care. Insights in these can help healthcare professionals in their communication with newly arrived migrants about this topic.

\section{Methods}

\section{Study design}

A qualitative research approach was used to explore Syrian refugees' perspectives and expectations with antibiotic use and prescribing in the Netherlands.

\section{Recruitment and sample}

Applying purposive and snowball sampling, Syrian refugees were recruited by using social media, thereby striving for diversity regarding age, gender and educational background. Recruitment went on until no new information was obtained and data saturation was reached.

Inclusion criteria were: a) being 18 years or older; b) living in the Netherlands with a refugee status, c) having consulted the general practitioner (GP) for minor illnesses or got/ tried to get antibiotics after coming to the Netherlands for themselves or for a young child (< 10 years),

When participants agreed to participate, they were informed about the study by phone, explaining the purpose of the study. When they gave verbal consent to be interviewed, the reasons of the study was again explained orally after which the participants signed the informed consent form. No relationships were built with the participants prior to the study.

\section{Data collection}


For the semi-structured interviews, an interview guide was developed with the following topics: 1) experiences with antibiotic use and prescribing within the Dutch health care system, 2) using antibiotics outside the Dutch health care system, 3) experiences with antibiotic use and prescribing in Syria, 4) awareness of antibiotic resistance, 5) negative and positive sides of the Dutch health care system and 6) negative and positive sides of the Syrian health care system.

The interviews were conducted in Arabic by researcher RA at the participants' homes in noise-free environment and were recorded using a mobile recording device; the interviews lasted between 35 and 47 minutes. Subsequently, all recordings were transcribed verbatim.

\section{Data analysis}

The transcripts were coded by the first author, (RA, female, conducted the interviews. At the time of data collection she was master's student and trained in qualitative study), double checked by another researcher (FdB, PhD and university teacher) using MAXQDA (www.maxqda, version 2018). The second step was to develop themes, whereby codes were clustered. Differences in coding and code clustering were discussed until agreement was reached. Afterwards, some of the research participants were asked for a member check, with the question whether they recognized the results. No changes were suggested.

\section{Results}

\section{Characteristics of respondents}

The characteristics of the participants are described in Table 1. Fourteen respondents were invited to participate in the study. Two of them declined because of the unfamiliarity and the lack of knowledge regarding research methods. Five males and nine females participated. They were between 30 and 46 years old. Most participants finished secondary school whereas four of them finished higher education. Before coming to the Netherlands, the participants were employed in various professions.

\section{Table 1. Respondent characteristics.}




\begin{tabular}{|llllll|}
\hline Respondent & Gender & Age & $\begin{array}{l}\text { Level of } \\
\text { education }\end{array}$ & $\begin{array}{l}\text { Former profession in } \\
\text { Syria }\end{array}$ & $\begin{array}{l}\text { Interview duration } \\
\text { (min.) }\end{array}$ \\
\hline R1 & Male & 40 & Secondary & Truck driver & 35 \\
\hline R2 & Male & 46 & Secondary & Pharmacist assistant & 40 \\
\hline R3 & Female & 31 & Secondary & Housewife & 47 \\
\hline R4 & Female & 32 & Higher & English teacher & 40 \\
\hline R5 & Female & 30 & Secondary & Nurse & 45 \\
\hline R6 & Male & 42 & Higher & Marketer & 35 \\
\hline R7 & Male & 35 & Higher & Pharmacist & 37 \\
\hline R8 & Male & 40 & Higher & Psychologist & 45 \\
\hline R9 & Female & 35 & Secondary & $\begin{array}{l}\text { Agriculture engineer } \\
\text { assistant }\end{array}$ & 35 \\
\hline R10 & Female & 31 & Secondary & Housewife & 44 \\
\hline R11 & Female & 42 & Higher & English teacher & 37 \\
\hline R12 & Female & 37 & Higher & Arabic teacher & 45 \\
\hline
\end{tabular}

Three main themes were derived from data analysis. Each theme consists of additional subthemes. The main themes were:

\section{Different practices in use and prescription of antibiotics in Syria and the Netherlands}

In Syria most respondents were used to getting antibiotics prescribed in case of minor illnesses, also for young children. However, some respondents explained that one does not always consult the doctor for a minor illness. It mainly takes place when the minor illness occurs in combination with additional symptoms which makes it difficult to diagnose oneself and to self-prescribe an antibiotic. When a person has the same symptoms, he or she tries to repeat the same medication using the previous prescription without re-consulting the doctor.

'Now I will explain how I acted in Syria. When the condition was more than usual, I consulted a doctor and when I had the same symptoms again, I went to the pharmacist to take the same antibiotic which the doctor prescribed the first time. For instance, when I had the flu, I went to the pharmacist to take antibiotics. But when I had additional symptoms such as vomiting, coughing or diarrhea I went to the doctor.'(female, 30 years old, nurse) 
All respondents mentioned that Syrians usually seek the advice of the pharmacist. They also are allowed to self-prescribe and buy antibiotics without an official prescription or consultation from the pharmacist. When a patient can pay more money, they often buy the most expensive antibiotic, which is perceived of as the best quality medication.

Some respondents reported not completing treatment courses. They stopped taking antibiotics once they felt better. Other respondents adhered to the instructions of completing antibiotic course.

When arriving to the Netherlands, Syrian refugees suddenly were confronted with a Dutch GP who declined to prescribe antibiotics for minor illnesses. Instead, the GP advised to drink warm tea or buy Paracetamol.

Since the dispensing of antibiotics without prescription is restricted in the Netherlands, half of the respondents accepted the advice of the GP and tried to use natural alternatives such as drinking (green) tea with lemon. A few respondents reported the adaptation to the new health care system, adhering to the GP's instructions regarding antibiotic use, completing the treatment course and not giving antibiotics to children without an official prescription.

However, the frustration with the GP service led one third of the patients to bring antibiotics in from outside the Netherlands or through a migrant network. The patients saved these antibiotics for emergencies and intended to self-medicate in case the GP refused to prescribe antibiotics to them.

'I have experienced a long time of tiredness and sickness until I contacted my family in Germany to send me antibiotics. From the first capsule, my health condition became much better.' (female, 31 years old, housewife)

\section{Health beliefs about the curative power of antibiotics}

The positive experiences with antibiotic use in Syria evoked belief in the curative power of antibiotics. Some participants had personal experience with the healing power of antibiotics and the necessity of antibiotics to recover.

Taking antibiotics was used by one respondent as prevention. He believed that one capsule of antibiotics daily can decrease the likelihood of being susceptible for an infection.

'Even if you are not sick, taking antibiotics, every day one pill, can prevent the illness. Therefore, in Syria, I used to take one pill daily or every two days.'(male, 40 years old, truck driver)

Most respondents considered that taking antibiotics could also be helpful for viral or influenza-like infections. Some of them believed that antibiotics are harmful for the body, so they stopped using antibiotics after feeling better. Others stressed the importance of completing the treatment course to recover completely and decrease the risk of falling ill again. Almost all respondents criticized the random use of antibiotics is Syria. 
'There is chaos and mess regarding antibiotic prescribing in Syria. They prescribe antibiotics for any minor illness. After living here, I realized that it is wrong and inefficient to do so.' (female, 35 years old, engineer assistant)

After having visited the Dutch GP, some Syrian refugees stated that it takes more time for them to recover when adhering to the suggested natural alternatives. For that reason, they rejected the natural alternatives and preferred antibiotics to have a shorter period of suffering from the illness.

I had flu one time after coming to the Netherlands and I did not take antibiotics. The duration of my illness was long. By taking antibiotics I feel that I recover quicker.'(male, 40 years old, psychologist).

Most respondents did not hear about the "antibiotic resistance" concept before. However, they became aware of it after coming in touch with the Dutch health care system. Some of them conceptualized antibiotic resistance as not being able to recover without taking antibiotics.

It was also evident that their health behavior was strongly influenced by their previous ways of dealing with illness. Therefore, they had difficulty in changing their behavior. Syrian refugees perceived having a weak immunity compared to Dutch persons since they started taking antibiotics from childhood on in Syria.

'I am trying to use antibiotics as they do in the Netherlands. However, our body immunity is different from theirs. For us, it is more difficult to reduce the use of antibiotics because we have been used to taking antibiotics from childhood on. Therefore, our bodies are not able to combat the illness without the aid of antibiotics.' (female, 31 years old, housewife)

Syrian refugees who believed that they had a vulnerable body immunity, which could not combat the illness, tried to find ways to self-medicate with antibiotics. Others started to reinforce their body immunity by not taking antibiotics, since they changed their perspective of using antibiotics after coming to the Netherlands. They also became more aware of the negative effects of antibiotics consumption. Some of them acknowledged resorting to self-medicating with antibiotics when feeling tired in order to alleviate the symptoms and to try to recover quicker.

On the other hand, mothers with young children adapted rapidly to the restricted regime in prescribing antibiotics in the Netherlands, because the children can overcome the obstacles of not prescribing antibiotics much easier than the adults.

'My children adapted to the Dutch health care system more easily than me because they are still children and their body immunity is still strong. For me, it is difficult to recover without antibiotics because I have been used to take it from childhood on.'(female, 37 years old, Arabic teacher)

Consequently, antibiotics were not given to the children without a prescription. Also the mothers perceived their children being less frequently sick and more able to recover from a minor illness without the aid of antibiotics. 


\section{Perspectives on two different health care systems}

The Syrian respondents showed a general sense of dissatisfaction with the Dutch health care system. The main issue was the difficult access to medication they desired. For example, antibiotics were not prescribed after an operation and instead a mild dose of Paracetamol was administered. This way of treatment made them feel that their ailments were not taken seriously. Another major complaint concerned the lack of professional knowledge they experienced in Dutch health care providers.

In the Netherlands, the doctor does not have the courage to diagnose your illness without the help of an apparatus. The GP has insufficient knowledge to treat you.' (female, 32 years old, English teacher)

The third point of criticism of the Dutch health care system concerned the difficulty of getting an immediate appointment with the GP. Therefore, respondents described how they exaggerated their symptoms and even lied about their symptoms in order to get an appointment with the GP.

'They do not give me an appointment if I do not have a fever for more than three days. I am always forced to lie and tell them that I have a fever for more than three days. In this way, I can make an appointment with the GP.' (female, 31 years old, housewife)

Finally, they also criticized the reluctance of Dutch GPs to refer patients to specialist are, which they cannot access without referral.

The participants emphasized that the GP should be aware of their cultural background and about the healthcare system they were used to. For instance, respondents mentioned the detailed questions doctors would ask them in Syria and the easy access to antibiotics within the Syrian health care system they prefer. The experience of having more freedom to choose the specialist and the instant help within the Syrian health care system appeared to be the main reasons for preferring the Syrian health care system.

I prefer the Syrian health care system because they have more professional knowledge than the Dutch doctors. In Syria, they help you instantly and you do not have to sustain that amount of pain to be helped afterwards. The specialists are available all the time.' (female, 42 years old, English teacher).

\section{Discussion}

This study highlights Syrian refugees' experiences with a new health care system that offers restricted access to antibiotics. On the one hand, this leads to the development of strategies to obtain antibiotics for self-medication. On the other hand, some of Syrian newcomers, especially mothers with young children, adhere to the new rules, because they feel their children have enough bodily strengths to combat the illness without antibiotics.

In general respondents were dissatisfied with Dutch General Practice as they felt not taken seriously, experienced difficulty to get prompt appointments and to get access to specialist care. 


\section{Comparison with other literature}

Despite the limited sample size and the particularity of the study population, some of the findings are plausible in the light of other studies. It has been confirmed that non prescribed antibiotics are administered over the counter by community pharmacies in Arab countries such as Syria, The United Arab Emirates and Kuwait [15-17]. Additionally, pharmacists in Syria do not perceive over the counter sales as a serious health problem for the patient [18].

More than the half of the Iraqi migrants in Jordan take antibiotics without a prescription [19]. Moreover, they believe that taking antibiotics is effective for viral infection [19], which runs parallel to our findings in which some Syrian migrants claim that antibiotics always help to cure viral infections.

A study in Saudi Arabia showed that about half of the participants stopped taking antibiotics once feeling better with the idea that no harmful effects would occur when not completing the treatment course of antibiotics [20].

Multiple studies refer to the behavior of migrants when coming from a country with easy access to antibiotics to a country with restricted access. In these studies, it is shown that migrants develop different strategies to deal with the restricted access to antibiotics.

Latinos in the United States self-medicate with antibiotics [21]. The same phenomenon was seen among Australian Chinese migrants, who obtain antibiotics from outside the health care system of their host country, store antibiotics at home and self-prescribe antibiotics [22]. Our findings showed the same home antibiotic storage among Syrian refugees in the Netherlands.

Another study among migrants in the United Kingdom reveals that some migrants bring antibiotics from their country of origin since they fail to adapt to the health care system in the new country where less antibiotics are prescribed. Others accept the new health care system and consequently adapt their health behavior [23]. Previous studies confirm the dissatisfaction with Dutch GP healthcare [24]. However, a recent study on Syrian migrants in the Netherlands reports that Syrian migrants are positive about the Dutch GP, who pays attention and gives advice to them [25]. That finding contradicts the perspectives as expressed by the Syrian respondents in this research in which they show discontent with the Dutch GP. A reason for this finding could be that the individual narratives give more room for an answer that is less socially desirable. Another reason for this finding could be being interviewed by a Syrian researcher. The respondents could have felt more at ease in expressing their feelings of dissatisfaction with the new health care system. According to the same report, nearly half of the respondents do not get the desired antibiotics [25], which runs parallel to our findings.

\section{Strengths and limitations}

To our knowledge, this article is the first to focus on Syrians' perspectives and expectations regarding antibiotic use and prescribing in Dutch primary care. Our findings emerged from the data, showing a variety of aspects on antibiotic use by Syrian migrants in the Netherlands which contributes to a better 
understanding of Syrians' health beliefs. In addition, the researcher has the same background as the participants, which could have led the respondents to feel more at ease in disclosing information about this topic, which can also be seen as a strength of this study.

A limitation is that study only relies on self-reports of the Syrian participants. No additional data, for instance from GP's, have been collected to give context to the perspectives of the Syrian participants. It also consists of a small sample of participants representing a certain age range (between 30 to 45 years old) with a relatively high educational level. The study lacks the perspectives of young adults and the elderly.

\section{Recommendations}

This study shows that more in-depth knowledge is needed to explore the strategies of newly arrived migrants and their complex reactions towards taking up a new health care system. Although there is a patient leaflet with information on antibiotic use in the Netherlands available in Arabic (eg., [26]), it cannot replace face to face information on this issue. Adult Syrian migrants should be persuaded to change their use of and perspectives on antibiotics, which is mainly due to their previous experiences with easy access and availability of antibiotics. Educational campaigns should include information about the appropriate administration and the meaningful benefits of antibiotics, the risks of easily prescribing antibiotics and the differences among health care systems in prescribing antibiotics. Additionally, action is required to improve the doctor-patient communication such as developing educational toolkits that consider various experiences of antibiotic use. That could equip the doctors better to work with migrants [23].

\section{Conclusion}

Syrian refugees experienced restricted access to antibiotics in Dutch primary care which was contrary to their experiences in Syria. As a reaction, they continued self-medicating with antibiotics. However, some of them adapted to the Dutch health care culture and accepted the alternative treatment proposal. For their children most participants adhered to the prescription policy of antibiotics in the Netherlands.

\section{Abbreviations}

WHO: World Health Organization

GP: General practitioner

\section{Declarations}

\section{Ethics approval and consent to participate}

The study protocol has been presented to the local Medical Ethics Committee. This study was classified as non-WMO by the Medical Ethics Committee (MEC), which means that no further ethical approval was 
required.

\section{Consent for publication}

Not applicable.

\section{Availability of data and materials}

The data consist of mainly transcripts of taped, in-depth interviews which cannot be shared because of privacy concerns and legal restrictions on data containing sensitive (health) information. Data are available from the corresponding author on reasonable request.

\section{Competing interests}

The authors have declared that no competing interests exist

\section{Funding}

The author(s) received no funding for this work.

\section{Authors' contributions}

RA contributed to the conception and design of the study, acquisition of the data, analyses and interpretation of the data, drafting of the manuscript, and gave final approval for submission of the manuscript. FdB contributed to the conception and design of the study, analyses and interpretation of the data, drafting of the manuscript, and gave final approval for submission of the manuscript. RK, EB, MVDM and SG contributed to the conception and design of the study, drafting of the manuscript, and gave final approval for submission of the manuscript. All authors have read and approved the manuscript

\section{Acknowledgments}

We are grateful to all participants who agreed to participate in this study.

\section{References}

1. Wise R, Hart T, Cars O, Streulens M, Helmuth R, Huovinen P, et al. Antimicrobial resistance: a major threat to public health. BMJ. 1998;:609-10.

2. World Health Organization. Antibiotic Resistance: Multi-Country Public Awareness Survey. WHO Press. 2015;:1-51.

3. Laxminarayan R, Duse A, Wattal C, Zaidi AKM, Wertheim HFL, Sumpradit N, et al. Antibiotic resistance-the need for global solutions. Lancet Infect Dis. 2013;13:1057-98.

4. Horne R, Graupner L, Frost S, Weinman J, Wright SM, Hankins M. Medicine in a multi-cultural society: The effect of cultural background on beliefs about medications. Soc Sci Med. 2004;59:1307-13. 
5. Hogenhuis CC, Grigoryan L, Me Numans M, Verheij TJM. Differences in antibiotic treatment and utilization of diagnostic tests in Dutch primary care between natives and non-western immigrants. Eur J Gen Pract. 2010;16:143-7.

6. Fatahi N, Krupic F. Factors Beyond the Language Barrier in Providing Health Care to Immigrant Patients. Med Arch. 2016;70:61-5.

7. Statstics Netherlands'. Population - migration background. 2020. https://opendata.cbs.nl/statline/\#/CBS/nl/dataset/37325/table?ts=1588339678243. Accessed 30 Apr 2020.

8. Phillimore J. Approaches to health provision in the age of super-diversity: Accessing the NHS in Britain's most diverse city. Crit Soc Policy. 2011;31:5-29.

9. Phillimore J. Delivering maternity services in an era of superdiversity: the challenges of novelty and newness. Ethn Racial Stud. 2015;38:568-82. doi:10.1080/01419870.2015.980288.

10. Esch T van, Mullenders P, Brabers A, Hek K, Jong J de. De rol van patiënten bij het afwijken van richtlijnen door huisartsen: een onderzoek naar het voorschrijven van antibiotica. | Nivel. Nivel. 2016. https://nivel.nl/sites/default/files/bestanden/Afwijken_van_richtlijnen.pdf. Accessed 1 Apr 2020.

11. Goossens H, Ferech M, Vander R, Md S, Goossens H, Ferech M, et al. For personal use. Only reproduce with permission from The Lancet Articles Introduction Outpatient antibiotic use in Europe and association with resistance: a cross-national database study. Lancet. 2005;365:579-87.

12. Barah F, Morris J, Goncalves V. Irrational use and poor public beliefs regarding antibiotics in developing countries: a pessimistic example of Syria. Int J Clin Pract. 2009;385 August:1263-4.

13. Welschen I, Kuyvenhoven M, Hoes A, Verheij T. Antibiotics for acute respiratory tract symptoms: Patients' expectations, GPs' management and patient satisfaction. Fam Pract. 2004;21:234-7.

14. Ashworth M, White P, Jongsma H, Schofield P, Armstrong D. Antibiotic prescribing and patient satisfaction in primary care in England: Cross-sectional analysis of national patient survey data and prescribing data. Br J Gen Pract. 2016;66:e40-6.

15. Barah F, Goncalves V. Antibiotic use and knowledge in the community in Kalamoon, Syrian Arab Republic: a cross-sectional study. East Mediterr Heal J. 2010;16:516-21.

16. Abasaeed A, Vlcek J, Abuelkhair M, Kubena A. Self-medication with antibiotics by the community of Abu Dhabi Emirate, United Arab Emirates. J Infect Dev Ctries. 2009;3:491-7.

17. Awad Al, Aboud EA. Knowledge, Attitude and Practice towards Antibiotic Use among the Public in Kuwait. PlosOne. 2015;10:1-15.

18. Al-Faham Z, Habboub G, Takriti F. The sale of antibiotics without prescription in pharmacies in Damascus, Syria. J Infect Dev Ctries. 2011;5:396-9.

19. Darwish DA, Abdelmalek S, Abu Dayyih W, Hamadi S. Awareness of antibiotic use and antimicrobial resistance in the Iraqi community in Jordan. J Infect Dev Ctries. 2014;8:616-23.

20. Hajjar W, Alnassar S, Al-khelb S, Al-mutairi S, Al-refayi N, Meo SA. Antibiotics use and misuse in upper respiratory tract infection patients: Knowledge, attitude and practice analysis in University Hospital , 
Saudi Arabia. J Pak Med Assoc. 2015;67:1387-92.

21. Larson E. Knowledge, attitudes, and practices regarding antibiotic use among Latinos in the United States: review and recommendations. Am J Infect Control. 2006;34:495-502.

22. Hu J, Wang Z. In-home antibiotic storage among Australian Chinese migrants. Int J Infect Dis. 2014;26:103-6. doi:10.1016/j.ijid.2014.04.017.

23. Lindenmeyer A, Redwood S, Griffith L, Ahmed S, Phillimore J. Recent migrants ' perspectives on antibiotic use and prescribing in primary care: $\mathrm{Br} J$ Gen Pract. 2016; November:802-9.

24. Teunissen E, Sherally J, Muijsenbergh M Van Den, Dowrick C, Weel-baumgarten E Van, Weel C Van. Mental health problems of undocumented migrants (UMs) in the Netherlands: a qualitative exploration of help-seeking behaviour and experiences with primary care. BMJ Open. 2014;:1-12.

25. Dagevos J, Huijnk W. Syriërs in Nederland. SCP. 2018; August.

https://www.scp.nl/publicaties/publicaties/2018/06/01/syriers-in-nederland. Accessed 1 Apr 2020.

26. Municipal Health Services (GGD-GHOR). http://www.ggdghorkennisnet.nl. Accessed 30 Apr 2020.

\section{Supplementary Files}

This is a list of supplementary files associated with this preprint. Click to download.

- Appendix.docx 